\title{
Synergistic Activity of Carfilzomib and Panobinostat in Multiple Myeloma Cells via Modulation of ROS Generation and ERK1/2
}

\author{
Lu Gao, Minjie Gao, Guang Yang, Yi Tao, Yuanyuan Kong, Ruixue Yang, Xiuqin Meng, \\ Gongwen Ai, Rong Wei, Huiqun Wu, Xiaosong Wu, and Jumei Shi \\ Department of Hematology, Shanghai Tenth People's Hospital, Tongji University School of Medicine, Shanghai 200072, China \\ Correspondence should be addressed to Xiaosong Wu; wux163@163.com and Jumei Shi; shijumei@hotmail.com
}

Received 13 August 2014; Accepted 31 August 2014

Academic Editor: Fenghuang Zhan

Copyright (c) $2015 \mathrm{Lu}$ Gao et al. This is an open access article distributed under the Creative Commons Attribution License, which permits unrestricted use, distribution, and reproduction in any medium, provided the original work is properly cited.

\begin{abstract}
Relapse of disease and subsequent resistance to established therapies remain as major challenges in the treatment of multiple myeloma (MM). New therapeutic options are needed for these extensively pretreated patients. To explore an optimized combinational therapy, interactions between the irreversible proteasome inhibitor carfilzomib exhibiting a well-tolerated sideeffect profile and histone deacetylase inhibitor (HDACi) panobinostat (LBH589) were examined in MM cells. Coadministration of carfilzomib and LBH589 led to a synergistic inhibition of proliferation in MM cells. Further studies showed that the combined treatment synergistically increased mitochondrial injury, caspase activation, and apoptosis in MM cells. Lethality of the carfilzomib/LBH589 combination was associated with the reactive oxygen species (ROS) generation and ERK1/2 inactivation. In addition, the free radical scavenger $\mathrm{N}$-acetylcysteine (NAC) could block carfilzomib and LBH589-induced oxidative stress and the subsequent apoptosis. Together, these findings argue that the strategy of combining carfilzomib and LBH589 warrants attention in MM.
\end{abstract}

\section{Introduction}

Multiple myeloma (MM) is a B-cell malignant disorder characterized by clonal proliferation of plasma cells in the bone marrow and osteolytic bone lesions [1]. Although new therapeutic options have been introduced and overall survival rate has improved in the management of MM, the disease remains incurable and almost all patients show disease relapse and develop drug resistance because of rapid regrowth of chemotherapy-refractory MM cells. This indicates that efficacious novel therapies are still needed for the patients with relapsed/refractory MM. Recently, data from present studies showed that the combination of proteasome inhibitor and histone deacetylase inhibitor (HDACi) resulted in synergistic inhibition of MM cell growth and might be an effective therapy for such patients $[2,3]$.

Carfilzomib, a second-generation selective, irreversible proteasome inhibitor of the chymotrypsin-like activity of the proteasome, shows antimyeloma effects [4]. Carfilzomib has been approved for the treatment of relapsed/refractory MM by the US Food and Drug Administration. It is well-tolerated in humans, especially a low incidence of peripheral neuropathy, and has activity against bortezomib-resistant myeloma cells, which makes it particularly suitable for use in combinational strategies [5]. Previous clinical trial has shown that replacing bortezomib with carfilzomib is safe and effective for MM patients failing bortezomib-containing combination regimens [6].

Panobinostat (LBH589), a highly potent HDACi, displays antitumor activity against a range of malignancies, particularly hematological diseases, such as MM, cutaneous T-cell lymphoma, Hodgkin lymphoma, and chronic myelogenous leukemia [7, 8]. LBH589 has shown activity against drugresistant cancer cell. LBH589 in combination with other therapies has shown synergistic antitumor efficacy by preclinical studies [8]. Some phase I/II clinical trials have been conducted to investigate the safety and efficacy of LBH589 in combination with other agents [9-11].

Present studies have demonstrated that the proteasome inhibitor bortezomib/HDACi combination has a powerful antimyeloma activity on MM cells including cells that are highly resistant to cytotoxic drugs $[12,13]$. Clinical trials 
further confirm such activity in relapsed/refractory MM patients $[3,9]$. However, a considerable part of patients in these clinical trials could not tolerate such therapy because of serious side effects and discontinued treatment [9]. This constrains its application to some extent and an optimized proteasome inhibitor/HDACi combination with lesser side effects is therefore needed. The second-generation proteasome inhibitor carfilzomib has a well-tolerated side-effect profile and potent antimyeloma activity. Thus, the carfilzomib/HDACi combination may represent an optimized proteasome inhibitor/HDACi combination therapy for MM patients if synergistic interactions between them exist. The purpose of the present study is to determine whether the combination of carfilzomib and LBH589 could have a synergistic activity on MM cells. Our results indicate that carfilzomib and LBH589 interact in a highly synergistic manner in all four tested MM cells and that events involve triggering reactive oxygen species (ROS) generation and inhibiting ERK1/2 pathway. Thus, our research provides a basis for clinical evaluation of this possible optimized combination of proteasome inhibitor and HDACi in relapsed/refractory MM patients.

\section{Materials and Methods}

2.1. Cells. The human MM cell lines RPMI 8226, OPM2, U266, and H929 were purchased from Cell Resource Center of Shanghai Institutes for Biological Sciences (Shanghai, China). Cells were maintained in RPMI-1640 medium (Invitrogen, Frederick, USA) containing 10\% fetal bovine serum, $1 \%$ penicillin (100 units $/ \mathrm{mL}$ ), and $1 \%$ streptomycin $(100 \mathrm{mg} / \mathrm{mL})$.

2.2. Reagents. Carfilzomib was purchased from Onyx Pharmaceuticals (South San Francisco, USA). LBH589 was purchased from Merck \& Co., Inc. (Rahway, USA). These agents were dissolved in dimethyl sulfoxide. N-Acetylcysteine (NAC) was purchased from Sigma-Aldrich (St. Louis, USA) and prepared in double-distilled water before use. Cell Counting Kit-8 (CCK-8) was purchased from Dojindo (Mashikimachi, Japan). Cell apoptosis kit was obtained from BD Pharmingen (Franklin Lakes, USA). JC-1 Mitochondrial Membrane Potential Assay kit was from Beyotime Institute of Biotechnology (Haimen, China).

2.3. Cell Survival Assay. MM cells were seeded into 96-well plates at a density of $2 \times 10^{5}$ cells per well and treated with different concentrations of carfilzomib and/or LBH589 for $48 \mathrm{~h}$. CCK- 8 was added into each well for an additional $2 \mathrm{~h}$ at $37^{\circ} \mathrm{C}$. The optical density was measured at $450 \mathrm{~nm}$ using a microplate reader and the cell survival rate was expressed as the absorbance relative to that of controls.

2.4. Assessment of Cell Apoptosis. After different drug treatments, RPMI 8226 cells were stained with Annexin V (BD Pharmingen, Franklin Lakes, USA) and propidium iodide (PI) (BD Pharmingen, Franklin Lakes, USA) according to the manufacturer's instructions. In our studies, the early apoptotic cells displayed Annexin $\mathrm{V}^{+} / \mathrm{PI}^{-}$staining and the late apoptotic cells displayed Annexin $\mathrm{V}^{+} / \mathrm{PI}^{+}$staining.

2.5. Analysis of Mitochondrial Membrane Potential. The changes in mitochondrial membrane potential $(\Delta \Psi \mathrm{m})$ were measured by flow cytometry using JC-1 staining according to the manufacturer's instructions. Briefly, RPMI 8226 cells were stained with 1 X JC-1 working solution for $20 \mathrm{~min}$ at $37^{\circ} \mathrm{C}$. Then cells were washed with JC-1 staining buffer and analyzed by flow cytometry.

2.6. Western Blot Analysis. Cells were lysed in lysis buffer (100 mM Tris-HCl, pH 6.8, 4\% SDS, 20\% glycerol) on ice for $30 \mathrm{~min}$. Proteins (30 $\mu \mathrm{g}$ ) were subjected to $10 \%$ or $12 \%$ SDS-PAGE and transferred to nitrocellulose membrane. The membranes were blocked with $5 \%$ bovine serum albumin for $1 \mathrm{~h}$ and probed with primary antibodies overnight at $4^{\circ} \mathrm{C}$, followed by treatment with appropriate secondary antibodies. Primary antibodies were as follows: caspase9, cleaved caspase-8, cleaved caspase-3, phospho-p44/42 MAPK (phospho-ERK1/2), p44/42 MAPK (ERK1/2), p38 mitogen-activated protein kinase, phospho-p38 mitogenactivated protein kinase, and $\beta$-actin antibodies. All were from Cell Signaling Technology (Beverly, USA).

2.7. Cell Cycle Distribution Analysis. Cells were collected and washed with ice cold phosphate buffered saline (PBS), fixed in $75 \%$ ethanol at $-20^{\circ} \mathrm{C}$ for $16 \mathrm{~h}$, and stained at $37^{\circ} \mathrm{C}$ for $15 \mathrm{~min}$ with PI containing $50 \mathrm{mg} / \mathrm{mL}$ RNase (BD Pharmingen, Franklin Lakes, USA) followed by flow cytometric analysis.

2.8. Measurement of ROS Generation. Cells were pretreated with or without NAC for $2 \mathrm{~h}$ at $37^{\circ} \mathrm{C}$ and then incubated with various drugs for indicated times. Then the cells were washed with PBS, resuspended in RPMI-1640 medium containing $10 \mu \mathrm{M}$ of $2^{\prime}, 7^{\prime}$-dichlorodihydrofluorescein diacetate (DCFHDA) (Beyotime, Haimen, China), and incubated at $37^{\circ} \mathrm{C}$ for $20 \mathrm{~min}$. Fluorescence intensity was assessed using a flow cytometer (BD, San Diego, USA).

2.9. Statistical Analysis. All data were expressed as mean \pm standard deviation (SD). Statistical significance of differences in multiple comparisons was determined by one-way ANOVA. $P<0.05$ was considered significant. Combination index (CI) was calculated using median dose effect analysis in conjunction with a commercially available software program (CalcuSyn, Biosoft).

\section{Results}

3.1. Concomitant Treatment with Carfilzomib and LBH589 Results in a Synergistic Inhibition of MM Cells Survival In Vitro. To assess what effect the combination of carfilzomib and LBH589 would have on MM cell survival, RPMI 8226, OPM2, U266, and H929 cells were incubated with increasing concentrations of carfilzomib and/or LBH589 for $48 \mathrm{~h}$, after which cytotoxicity was evaluated by CCK-8 assay. As shown in Figure 1(a), compared to individual exposure (excepting 

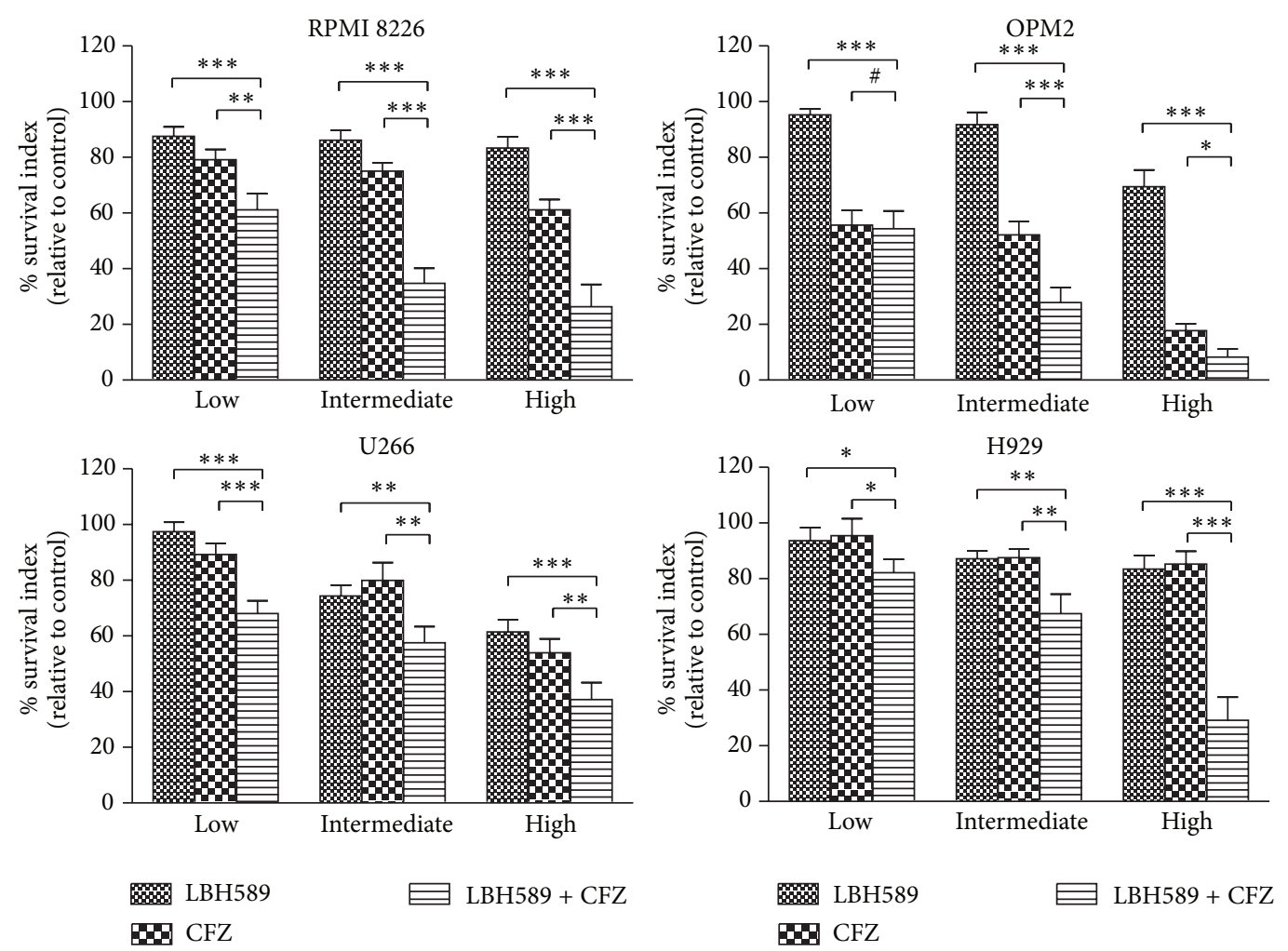

(a)
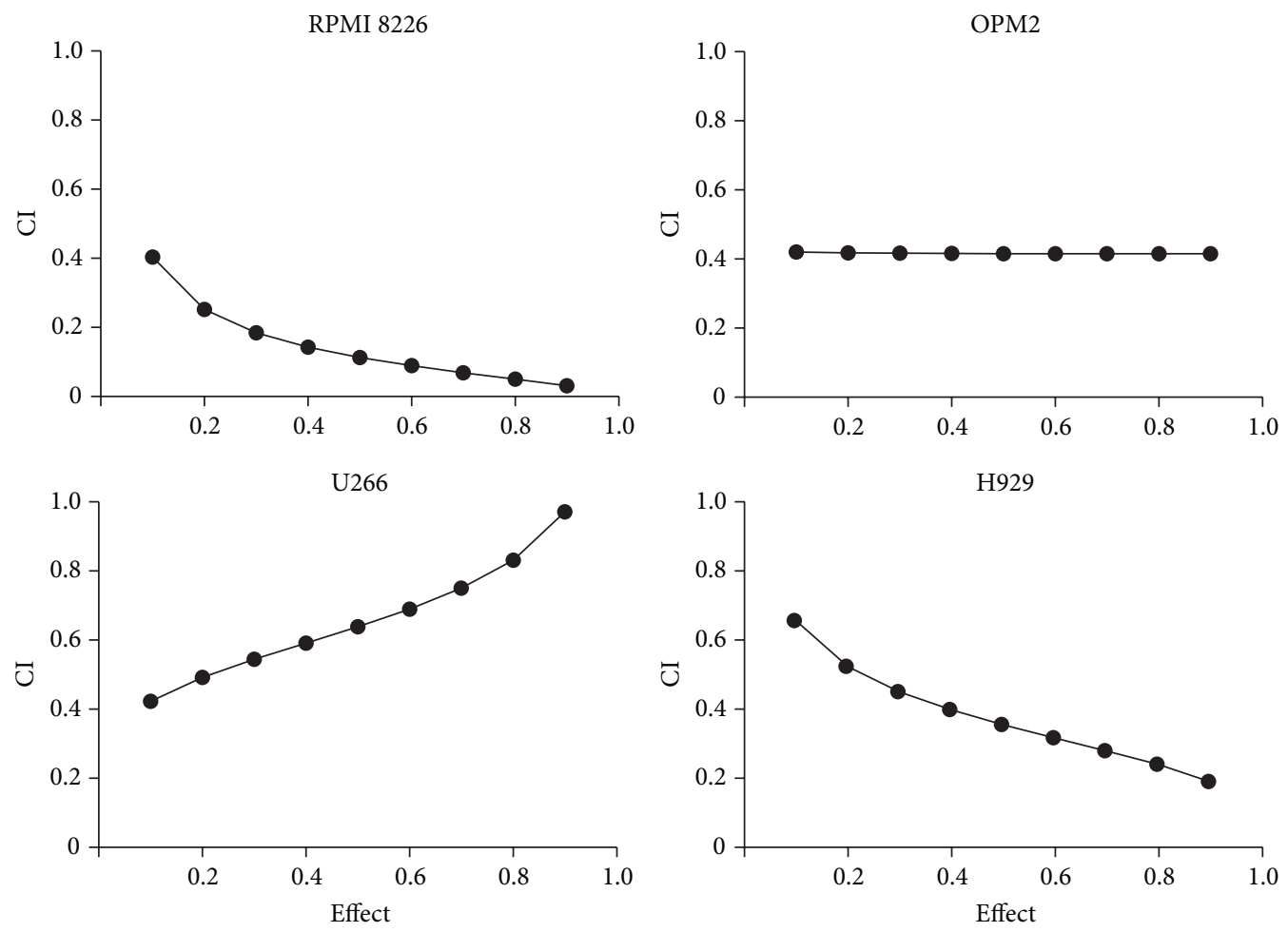

(b)

FIGURE 1: Coadministration of carfilzomib and LBH589 induced a synergistic inhibition of proliferation in MM cells. (a) All cell lines were incubated for $48 \mathrm{~h}$ with carfilzomib and/or LBH589 (low: $2 \mathrm{nM} \mathrm{LBH589,} 20 \mathrm{nM}$ carfilzomib; intermediate: 4 nM LBH589, 40 nM carfilzomib; and high: $6 \mathrm{nM} \mathrm{LBH589,} 60 \mathrm{nM}$ carfilzomib) followed by CCK-8 assay. Data represent the mean \pm SD for three separate experiments performed in triplicate. ${ }^{*} P<0.05 .{ }^{* *} P<0.01 .{ }^{* * *} P<0.001 .{ }^{*} P>0.05$. CFZ, carfilzomib. (b) CI values were calculated using median dose effect analysis. CI values $<1.0$ denote synergistic interactions. 

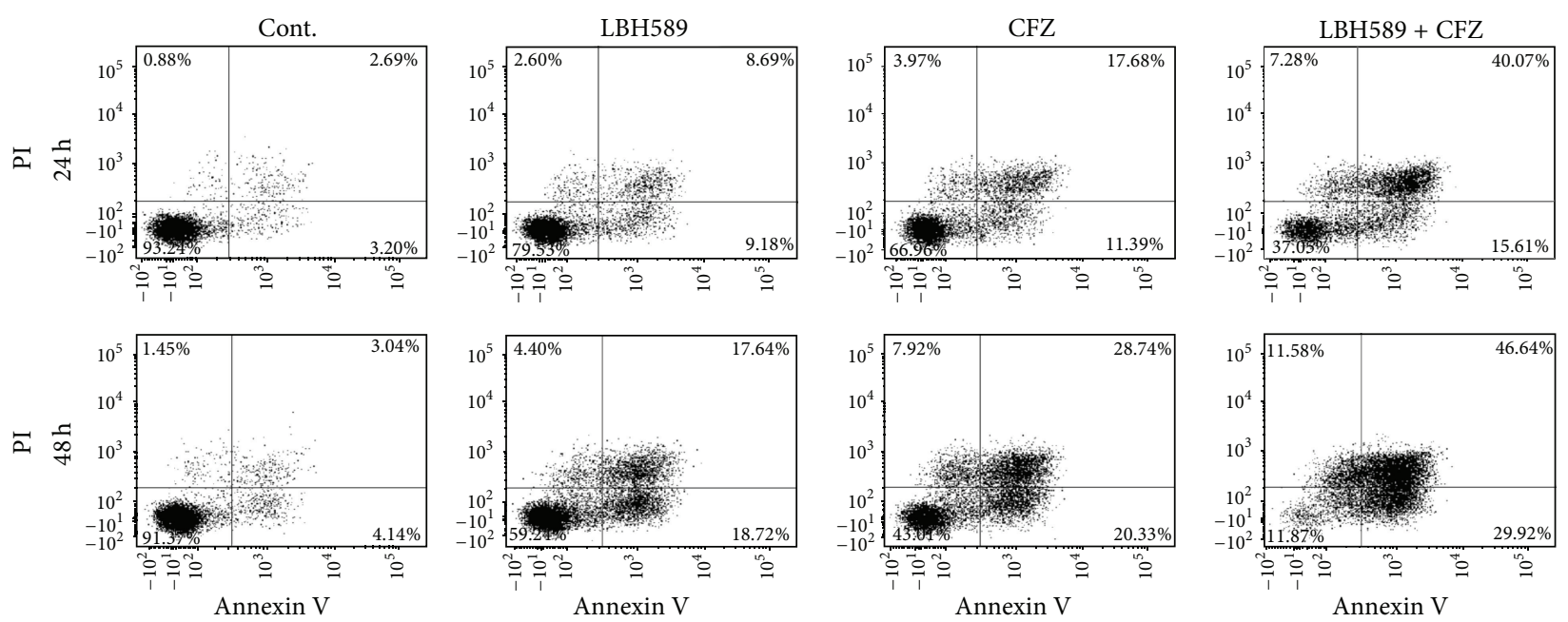

FIGURE 2: Combination of carfilzomib and LBH589 induced enhanced apoptosis in MM cells. RPMI 8226 cells were exposed to carfilzomib ( $40 \mathrm{nM}$ ) and/or LBH589 (4 nM) for $24 \mathrm{~h}$ (upper panel) or $48 \mathrm{~h}$ (low panel) followed by Annexin V-FITC/PI double staining and flow cytometry analysis. The percentage of apoptotic cells ( $24 \mathrm{~h})$ in Cont., LBH589, CFZ, and LBH589 + CFZ group was $6.7 \% \pm 0.9 \%, 18.8 \% \pm 2.8 \%, 30.0 \% \pm$ $3.5 \%$, and $56.6 \% \pm 8.8 \%{ }^{*}$, respectively. The value $(48 \mathrm{~h})$ was $6.8 \% \pm 1.5 \%, 35.9 \% \pm 3.8 \%, 48.3 \% \pm 3.8 \%$, and $81.5 \% \pm 5.0 \%{ }^{*}$, respectively. ${ }^{*} P<0.05$ versus control group; $n=3$. The data shown are representative of three independent experiments. Cont., control. CFZ, carfilzomib.

individual exposure of OPM2 cells to low concentration of carfilzomib), combined exposure to low, intermediate, and high concentrations of carfilzomib and LBH589 induced a more significant decrease in the growth of all four tested MM cell lines $(P<0.05)$. The median dose effect analysis for all tested MM cells exposed to carfilzomib and LBH589 yields CI values which were substantially less than 1.0, indicating a synergistic interaction (Figure 1(b)).

3.2. Concomitant Treatment with Carfilzomib and LBH589 Effectively Induces Apoptosis, Mitochondrial Injury, and Caspase Activation in MM Cells. Annexin V/PI double staining was performed to determine the apoptosis of RPMI 8226 cells exposed to carfilzomib ( $40 \mathrm{nM})$ and/or LBH589 ( $4 \mathrm{nM})$ for $24 \mathrm{~h}$ or $48 \mathrm{~h}$. Compared with the control, individual treatment with LBH589 only caused a moderate increase in the percentage of Annexin $\mathrm{V}^{+}$cells $(18.8 \% \pm 2.8 \%$ versus $6.7 \% \pm 0.9 \%$ for $24 \mathrm{~h}$ and $35.9 \% \pm 3.8 \%$ versus $6.8 \% \pm$ $1.5 \%$ for $48 \mathrm{~h}$ ). The values were relatively high in carfilzomib treated RPMI 8226 cells $(30.0 \% \pm 3.5 \%$ for $24 \mathrm{~h}$ and $48.3 \% \pm$ $3.8 \%$ for $48 \mathrm{~h}$ ). On the other hand, MM cells exposed to carfilzomib and LBH589 exhibited a much higher percentage of Annexin $\mathrm{V}^{+}$cells $(56.6 \% \pm 8.8 \%$ for $24 \mathrm{~h}$ and $81.5 \% \pm$ $5.0 \%$ for $48 \mathrm{~h}$ ), indicating that the combination resulted in a significant induction of apoptosis (Figure 2).

The reduction of $\Delta \Psi \mathrm{m}$ is a vital event in the initiation of apoptotic cascade. The combined treatment of carfilzomib $(40 \mathrm{nM})$ and LBH589 $(4 \mathrm{nM})$ induced $35.0 \% \pm 3.0 \%$ loss of $\triangle \Psi \mathrm{m}$ in RPMI 8226 cells at $24 \mathrm{~h}$, as represented by the cells with decreased JC-1 red fluorescence, whereas the loss of $\Delta \Psi \mathrm{m}$ was only $20.3 \% \pm 4.0 \%$ for carfilzomib $(40 \mathrm{nM})$ and $16.3 \% \pm 2.9 \%$ for LBH589 $(4 \mathrm{nM})$ at $24 \mathrm{~h}$. Moreover, a more significant loss of $\Delta \Psi \mathrm{m}$ was observed in the combinational treatment $(62.4 \% \pm 5.0 \%)$ compared with those treated with carfilzomib $(40.1 \% \pm 4.6 \%)$ or LBH589 $(20.8 \% \pm 3.1 \%)$ alone at $48 \mathrm{~h}$ (Figure 3(a)). To further confirm that the combined treatment with carfilzomib and LBH589 did trigger classical apoptosis in MM cells, caspase activation, another pivotal event associated with the activation of apoptotic cell death, was examined by Western blot analysis. As shown in Figure 3(b), a clear cleavage of caspase-8, caspase-9, and caspase-3 was observed in RPMI 8226 cells after incubation with both carfilzomib (40 nM) and LBH589 (4 nM) for $24 \mathrm{~h}$. In contrast, only modest cleavage of all three caspase proteins was detected in RPMI 8226 cells after 24-hour treatment of carfilzomib $(40 \mathrm{nM})$ or LBH589 (4nM) alone. These findings indicate that combined treatment of MM cells with carfilzomib and HDACi LBH589 potently induces $\Delta \Psi \mathrm{m}$ loss and caspase activation, events associated with activation of the apoptotic program.

3.3. The Effects of Carfilzomib and/or LBH589 on Cell Cycle Distribution. Cell cycle analysis was performed in U266 cells exposed to carfilzomib ( $40 \mathrm{nM}$ ) and/or LBH589 (4 nM) for $24 \mathrm{~h}$. Compared with the control, treatment with carfilzomib resulted in $\mathrm{G}_{1}-\mathrm{G}_{0}$ arrest accompanied by a decrease in $S$ phase cell population $(n=3, P<0.01)$. Individual treatment with LBH589 had little effect on cell cycle distribution. Similarly, neither $G_{1}-G_{0}$ arrest nor $G_{2}-M$ arrest was observed in combined treatment $(n=3, P>0.05)$ despite the synergistic induction of apoptosis caused by combined treatment (Figure 4). Similar results were observed in RPMI 8226 cells after treatment with carfilzomib $(40 \mathrm{nM})$ and/or LBH589 ( $4 \mathrm{nM})$ for $24 \mathrm{~h}$ (data not shown).

3.4. Combined Exposure of MM Cells to Carfilzomib and LBH589 Induces ROS Generation. Previous studies have reported that cytotoxicity induced by bortezomib/HDACi 

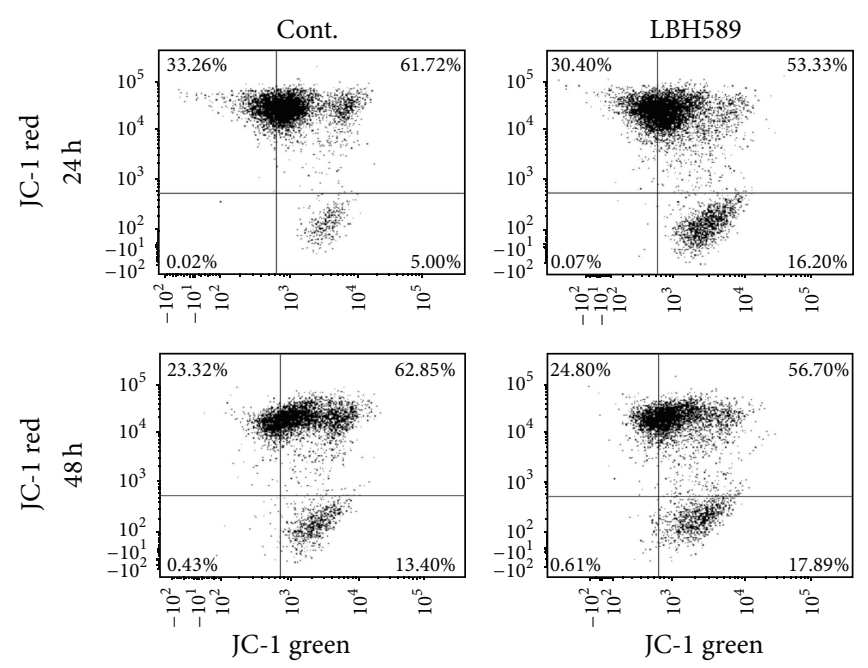

(a)
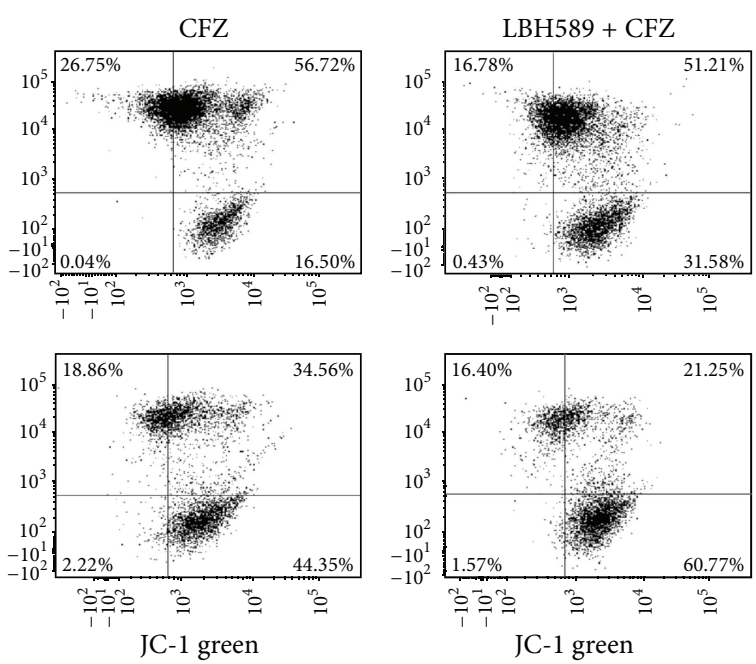
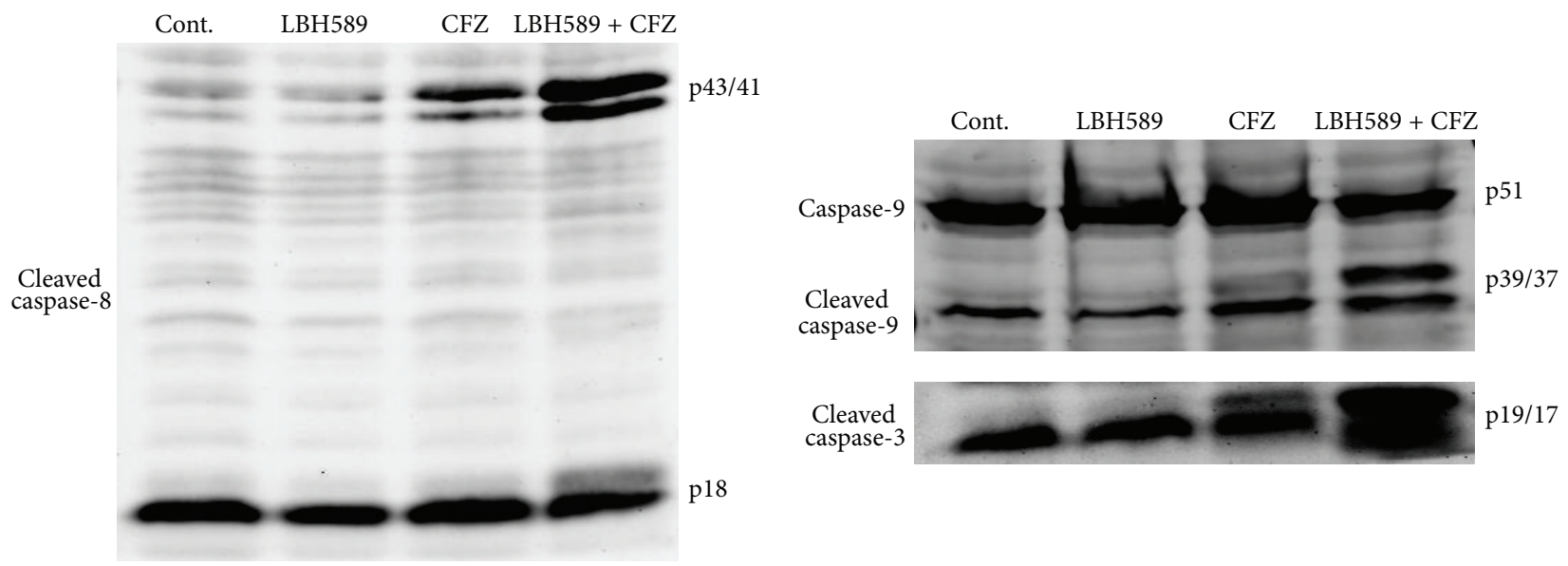

(b)

FIGURE 3: Concomitant treatment with carfilzomib and LBH589 synergistically resulted in mitochondrial injury and caspase activation. (a) RPMI 8226 cells were treated with carfilzomib $(40 \mathrm{nM})$ and/or LBH589 $(4 \mathrm{nM})$ for $24 \mathrm{~h}$ (upper panel) or $48 \mathrm{~h}$ (low panel), after which JC-1 staining was performed. $\Delta \Psi \mathrm{m}$ was assessed by flow cytometry. Only JC-1 green positive (lower right quadrant) cells were analyzed for the loss of $\Delta \Psi \mathrm{m}$. The loss of $\Delta \Psi \mathrm{m}(24 \mathrm{~h})$ in Cont., LBH589, CFZ, and LBH589 + CFZ group was $5.3 \% \pm 2.1 \%, 16.3 \% \pm 2.9 \%, 20.3 \% \pm 4.0 \%$, and $35.0 \% \pm$ $3.0 \%{ }^{* * *}$, respectively. The value $(48 \mathrm{~h})$ was $12.2 \% \pm 3.5 \%, 20.8 \% \pm 3.1 \%, 40.1 \% \pm 4.6 \%$, and $62.4 \% \pm 5.0 \%{ }^{* * *}$, respectively. ${ }^{* * *} P<0.001$ versus control group; $n=3$. (b) RPMI 8226 cells were treated with carfilzomib (40 nM) and/or LBH589 (4 nM) for 24 h. Then, caspase-9 (p51) and cleaved caspase-8 (p43/41, p18), caspase-9 (p39/37), and caspase-3 (p19/17) were monitored by Western blot analysis. Cont., control. CFZ, carfilzomib.

combination originates from ROS generation $[2,14]$. Studies were therefore performed to investigate whether such mechanism is also responsible for carfilzomib and LBH589induced cytotoxicity. As shown in Figure 5(a), individual treatment with carfilzomib $(40 \mathrm{nM})$ or LBH589 $(4 \mathrm{nM})$ had modest effect on ROS levels in RPMI 8226 cells, whereas the combined treatment resulted in a marked increase in ROS generation, which was substantially abrogated by the free radical scavenger NAC $(15 \mathrm{mM})$. In addition, 9 hours of combined treatment with carfilzomib (40 nM) and LBH589 $(4 \mathrm{nM})$ induced the most obvious ROS generation in RPMI 8226 cells (Figure 5(b)). To assess the importance of ROS generation in carfilzomib and LBH589-induced apoptosis, RPMI 8226 cells were preincubated with NAC for $2 \mathrm{~h}$ and then treated with carfilzomib (40 nM) and LBH589 $(4 \mathrm{nM})$ for $24 \mathrm{~h}$. As expected, NAC significantly $(P<0.001)$ reduced the apoptosis induced by the combined treatment (Figure 5(c)), suggesting that ROS generation plays an important role in carfilzomib and LBH589-mediated cytotoxicity in MM cells.

3.5. Synergistic Induction of Apoptosis after Combined Treatment Involves the ERK1/2 Pathway. To determine the molecular mechanisms underlying the carfilzomib/LBH589 lethality in MM cells, several relevant signaling pathways were investigated. As shown in Figure 5(d), compared with the control, exposure of RPMI 8226 cells to carfilzomib ( $40 \mathrm{nM}$ ) and LBH589 ( $4 \mathrm{nM}$ ) for $24 \mathrm{~h}$ markedly decreased the level of 


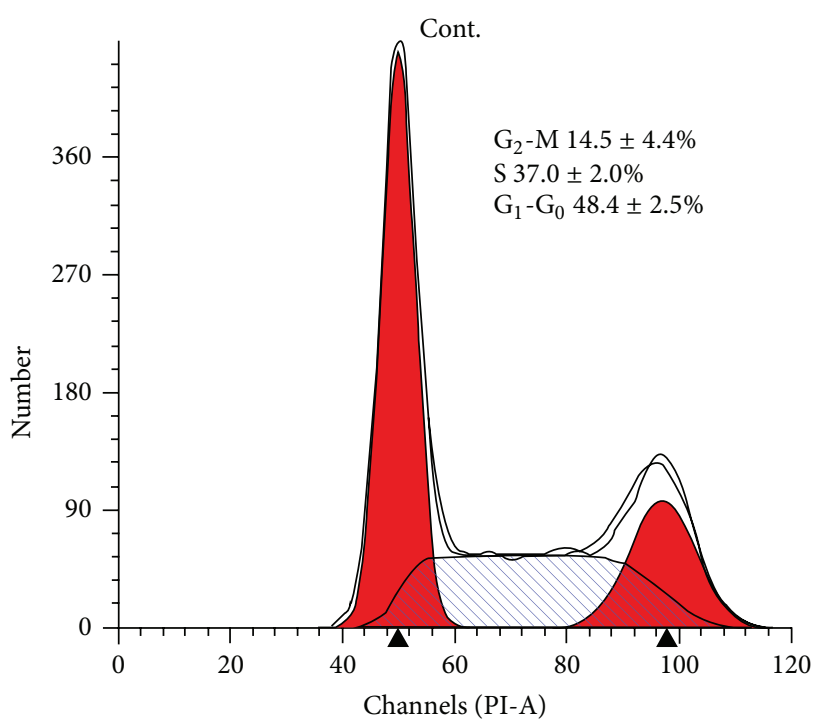

(a)

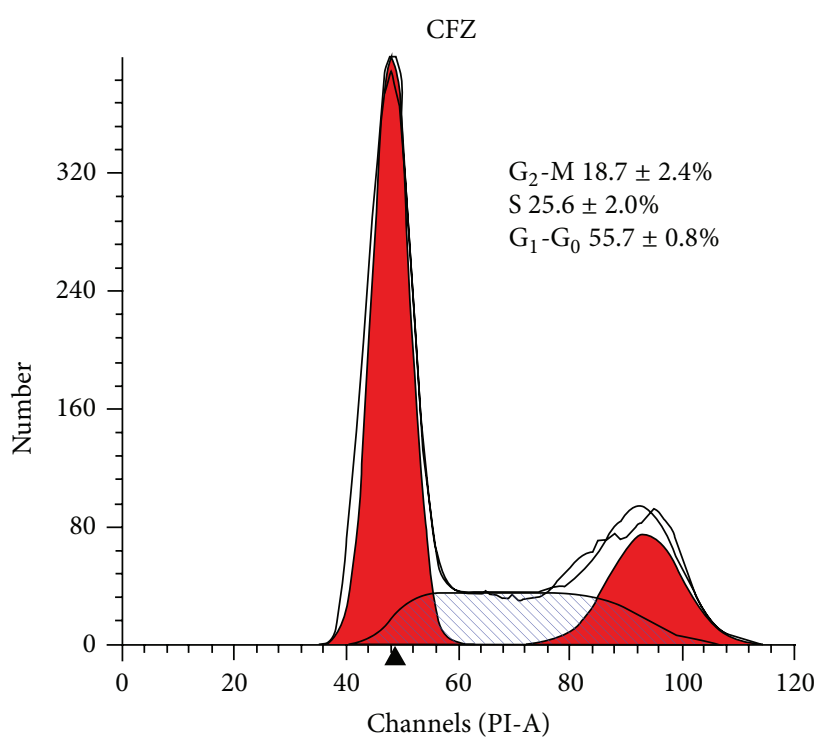

(c)

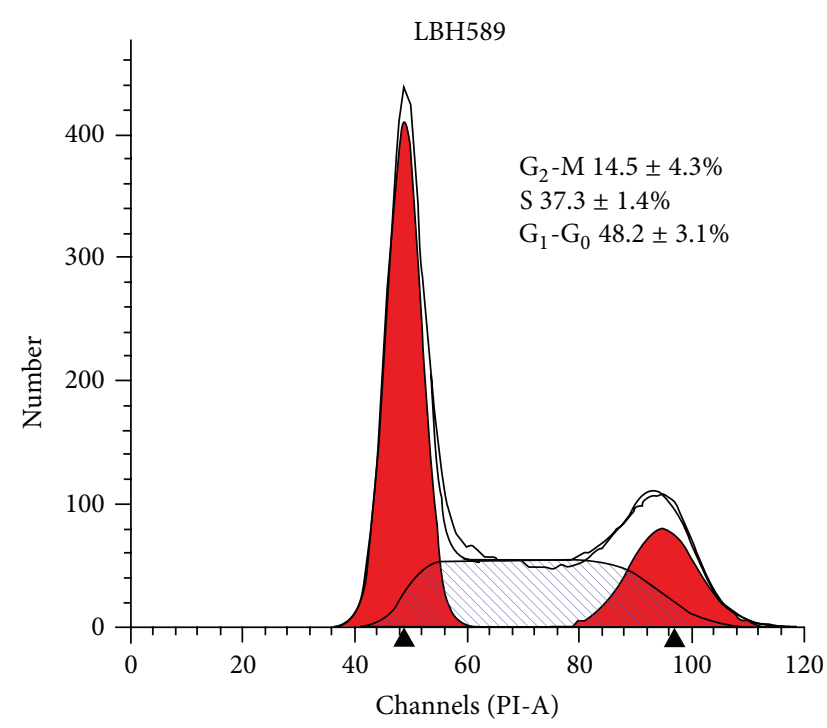

(b)

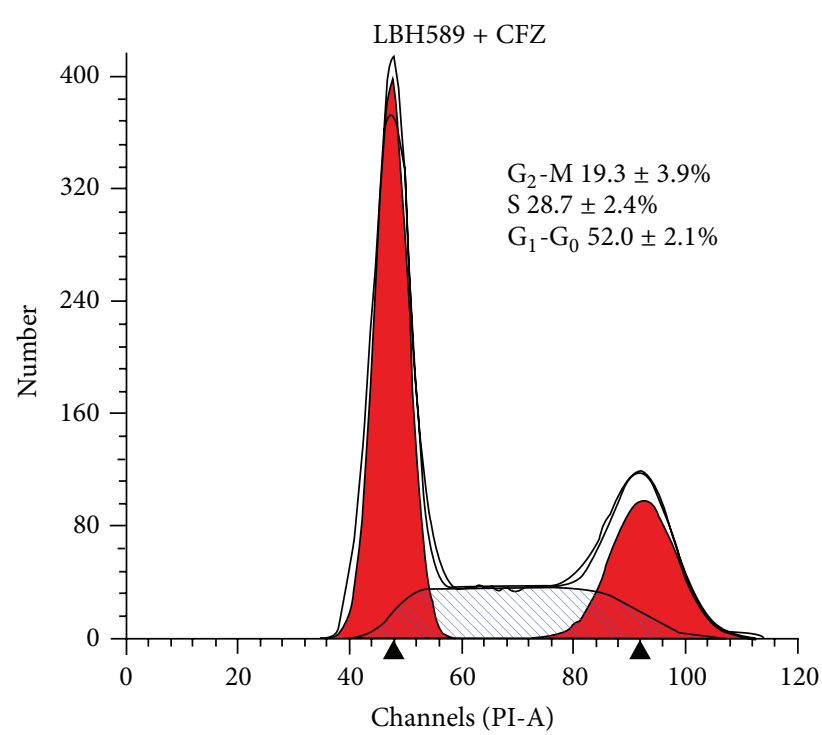

(d)

FIGURE 4: Concomitant treatment with carfilzomib and LBH589 showed no effect on MM cell cycle distribution. MM cells were treated with carfilzomib (40 nM) and/or LBH589 (4 nM) for $24 \mathrm{~h}$ followed by PI staining and flow cytometry analysis. Data represent the mean \pm SD for three separate experiments performed in triplicate. Only the percentage of $G_{1}-G_{0}$ in $C F Z$ group was significantly different from that in control group $(P<0.01)$. The percentage of $\mathrm{G}_{1}-\mathrm{G}_{0}$ in $\mathrm{LBH} 589$ and $\mathrm{LBH} 589+\mathrm{CFZ}$ group and $\mathrm{G}_{2}-\mathrm{M}$ in CFZ, LBH589, and LBH589 + CFZ group was similar to that in control group $(P>0.05)$. Cont., control. CFZ, carfilzomib.

ERK1/2 phosphorylation without obvious changes in the total ERK levels, suggesting that the ERK1/2 pathway, protecting MM cells from apoptosis, was effectively inhibited by the combined treatment. Carfilzomib (40 nM) or LBH589 (4 nM) alone had no significant effect on the phosphorylation of ERK1/2 and total ERK levels. A similar profile was observed in OPM2 cells. In the present study, p38MAPK signaling pathway, another major mechanism involved in the modulation of MM cell apoptosis, was also analyzed. As shown in Figure 5(d), the levels of total p38 and the p38 phosphorylation were much alike amongst different treatments.

\section{Discussion}

Combinational therapies, with agents that are synergistic when combined, are often required for patients with relapsed and/or refractory MM [15]. Among them, bortezomib/HDACi combination attracts the most attention because of powerful antimyeloma activity. However, side effects of bortezomib/HDACi combination observed in clinical trials confine its application in some MM patients [9]. In the present study, we examine the interactions between carfilzomib and HDACi LBH589 to explore a possible optimized 


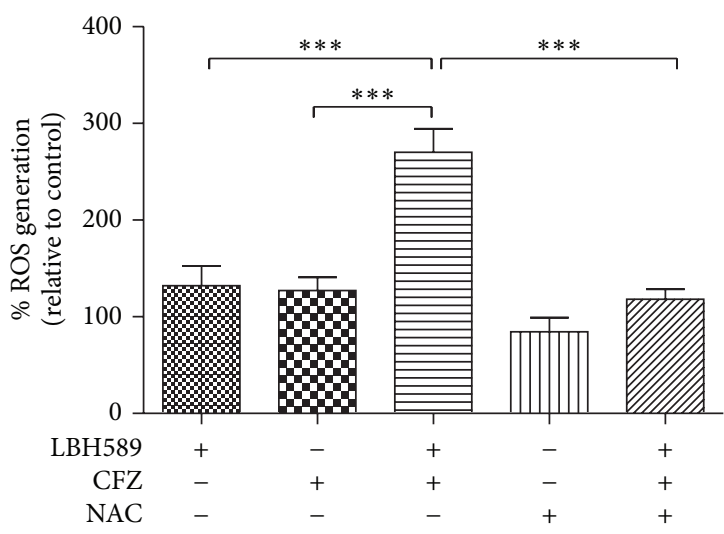

(a)
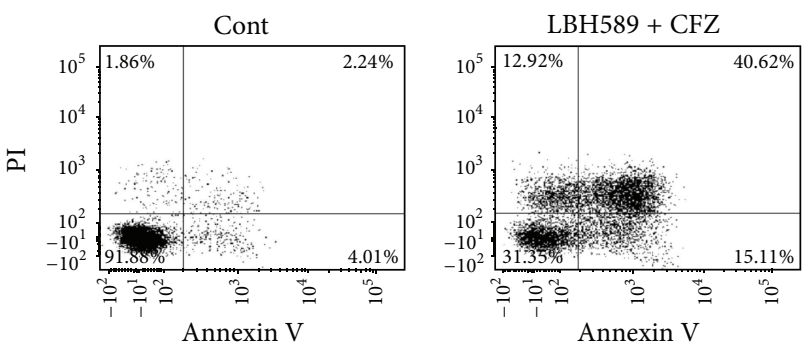

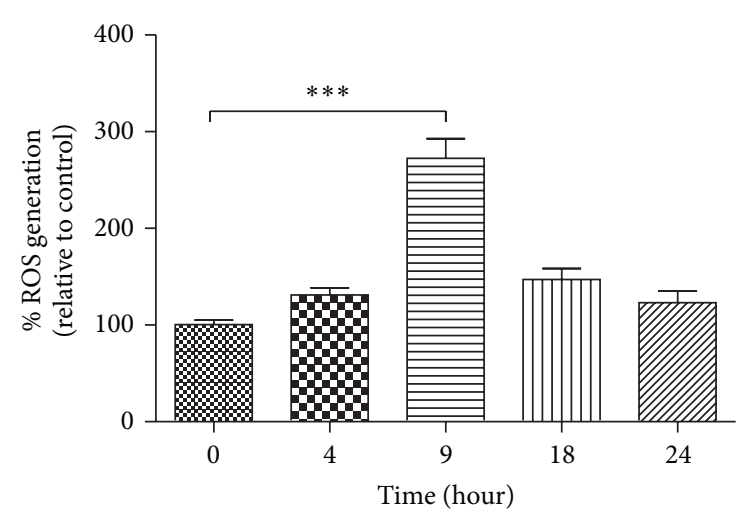

(b)
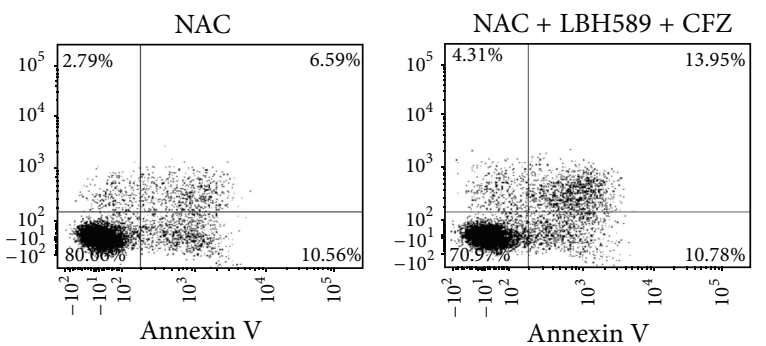

(c)
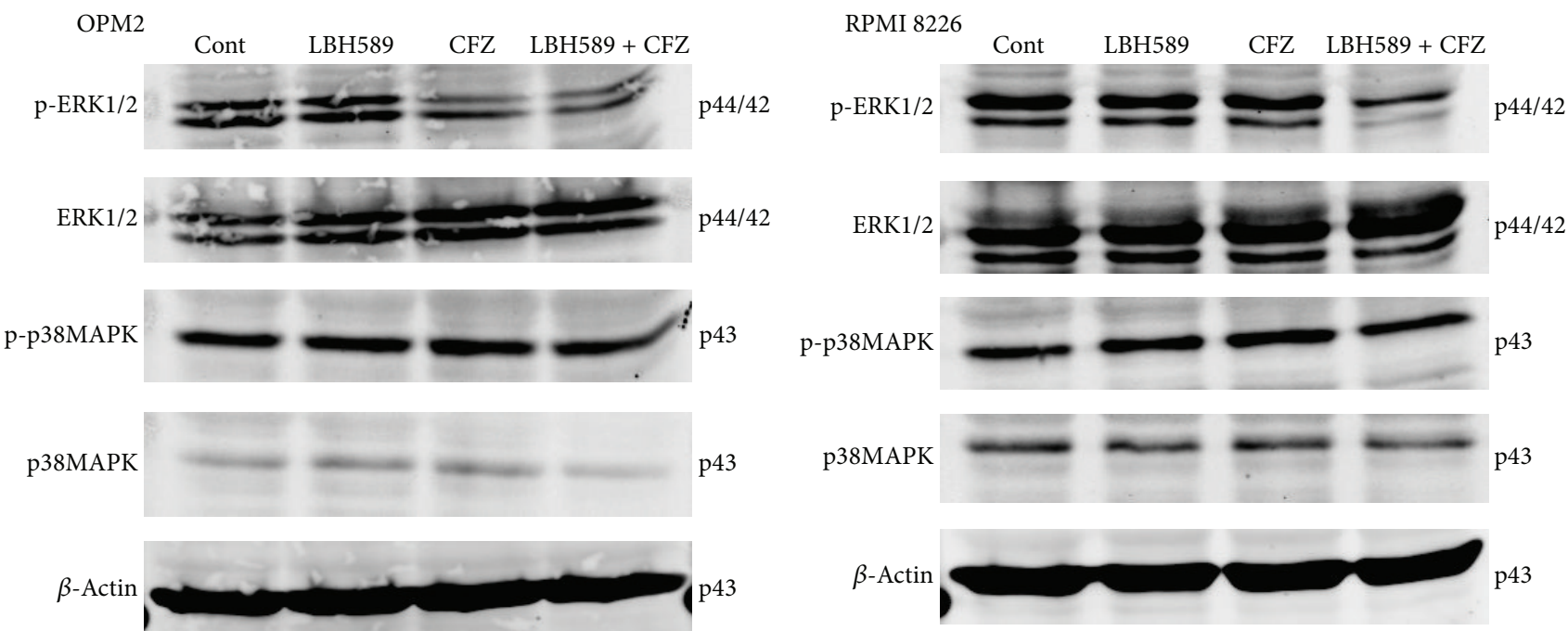

(d)

FIGURE 5: Lethality of the carfilzomib/LBH589 combination in MM cells was associated with the ROS generation and ERK1/2 inactivation. (a) RPMI 8226 cells were pretreated with or without NAC for $2 \mathrm{~h}$ at $37^{\circ} \mathrm{C}$ and then incubated with carfilzomib ( $\left.40 \mathrm{nM}\right)$ and/or LBH589 ( $4 \mathrm{nM}$ ) for $24 \mathrm{~h}$, after which ROS generation was detected. ${ }^{* * *} P<0.001 . n=3$. (b) ROS generation in RPMI 8226 cells was monitored 0 , 4, 9, 18, and 24 hours after combined treatment with carfilzomib $(40 \mathrm{nM})$ and LBH589 $(4 \mathrm{nM}) .{ }^{* * *} P<0.001 . n=3$. (c) RPMI 8226 cells were pretreated with or without NAC for $2 \mathrm{~h}$ at $37^{\circ} \mathrm{C}$ and then incubated with or without carfilzomib ( $40 \mathrm{nM}$ ) and LBH589 (4 nM) for $24 \mathrm{~h}$. Apoptosis rate in Cont., LBH589 + CFZ, NAC, and NAC + LBH589 + CFZ group was 6.5\% $\pm 1.9 \%, 55.3 \% \pm 9.2 \%, 12.4 \% \pm 4.2 \%$, and $29.2 \% \pm 4.3 \%{ }^{* * *}$, respectively. ${ }^{* * *} P<0.001$ versus LBH589 + CFZ group. $n=3$. The data shown are representative of three independent experiments. (d) OPM2 and RPMI 8226 cells were treated with carfilzomib (40 nM) and/or LBH589 (4 nM) for $24 \mathrm{~h}$. Then, Western blot analysis of ERK1/2, p-ERK1/2, p38MAPK, and p-p38MAPK was performed. The levels of $\beta$-actin were used as the loading control. Cont., control. CFZ, carfilzomib. 
combinational therapy of proteasome inhibitor and HDACi for MM.

We observed a synergistic inhibition of cell proliferation and apoptosis in MM cells after combined treatment with carfilzomib and LBH589. To further confirm the apoptosis, $\Delta \Psi \mathrm{m}$ loss and caspase cleavage, events associated with apoptosis activation, were investigated. Our data showed that the levels of cleaved caspase- 9 , caspase- 8 , and caspase- 3 were markedly increased in MM cells exposed to carfilzomib and LBH589 compared with single drug treatment, suggesting that both intrinsic (caspase-9) and extrinsic (caspase-8) apoptotic pathways were activated after combined treatment. Moreover, the more loss of $\Delta \Psi \mathrm{m}$ in both drugs treated cells further indicated the activation of intrinsic apoptotic pathway.

Since cell cycle arrest is often associated with apoptosis and several studies have shown that the proteasome inhibitor/HDACi combination, inducing obvious apoptosis, caused $G_{1}-G_{0}$ or $G_{2}-M$ arrest $[13,14]$, cell cycle analysis was performed in the present study. Our data showed that carfilzomib alone induced $\mathrm{G}_{1}-\mathrm{G}_{0}$ arrest in $\mathrm{MM}$ cells, whereas no significant changes in cell cycle distribution were observed after individual treatment with LBH589 or combined treatment. The result was consistent with another study in which the proteasome inhibitor/HDACi combination also failed to induce $G_{1}-G_{0}$ or $G_{2}-M$ arrest [16]. Reasons for the discrepancy in these studies are unclear, but variations in cell types and different doses of the combined drugs may be responsible for the observed difference.

Previous studies in various tumor cells have indicated that bortezomib or HDACi-induced lethality is related to ROS generation [17-20]. Moreover, lethal effects induced by the combined treatment with proteasome inhibitor and HDACi in leukemia and lymphoma cells have also been demonstrated to proceed through a ROS-dependent mechanism [21, 22]. In the present study, we showed that combined treatment with carfilzomib and LBH589 induced a marked increase in ROS in MM cells and the free radical scavenger NAC attenuated the oxidative stress, as well as the subsequent apoptosis. Thus, our data provide further support for the notion that ROS generation is a crucial factor in proteasome inhibitor/HDACi-mediated lethality.

Activation of ERK1/2 pathway has been shown to protect malignant cells from the lethality of oxidative stress [23] and therefore confers a survival advantage on these cells. Studies have indicated that apoptosis induction of several antitumor drugs, alone or in combination, is associated with inactivation of this cytoprotective pathway. For example, inhibition of ERK1/2 pathway is one of the molecular mechanisms underlying lethality of bortezomib and HDACi combination on T-leukemia/lymphoma cells [24]. In our present study, when carfilzomib was combined with LBH589, a significant decrease in the level of ERK1/2 phosphorylation was observed, whereas phosphorylation of p38 exhibited no change. This suggests that inhibition of the ERK1/2 pathway may be the right mechanism through which carfilzomib and LBH589 combination induces apoptosis.

In summary, our data indicate that carfilzomib and LBH589 combination synergistically induces apoptosis in
MM cells, which is accomplished by enhancing ROS generation and decreasing ERK1/2 phosphorylation. Thus, we provide a basis for clinical evaluation of carfilzomib/LBH589 combination in relapsed/refractory MM patients.

\section{Conflict of Interests}

The authors declare that there is no conflict of interests.

\section{Authors' Contribution}

Lu Gao, Minjie Gao, and Guang Yang contributed equally to this work.

\section{Acknowledgments}

This study was supported by Grants from the National Natural Science Foundation of China (nos. 81372391, 81071856, and 81228016), Shanghai Science and Technology Program (no. 12410705100), and Shanghai Tenth People's Hospital Funds (no. 040113015).

\section{References}

[1] S. Genadieva-Stavric, F. Cavallo, and A. Palumbo, "New approaches to management of multiple myeloma," Current Treatment Options in Oncology, vol. 15, no. 2, pp. 157-170, 2014.

[2] X.-Y. Pei, Y. Dai, and S. Grant, "Synergistic induction of oxidative injury and apoptosis in human multiple myeloma cells by the proteasome inhibitor bortezomib and histone deacetylase inhibitors," Clinical Cancer Research, vol. 10, no. 11, pp. 38393852, 2004.

[3] D. M. Weber, T. Graef, M. Hussein et al., "Phase i trial of vorinostat combined with bortezomib for the treatment of relapsing and/or refractory multiple myeloma," Clinical Lymphoma, Myeloma and Leukemia, vol. 12, no. 5, pp. 319-324, 2012.

[4] K. Fostier, A. de Becker, and R. Schots, "Carfilzomib: a novel treatment in relapsed and refractory multiple myeloma," OncoTargets and Therapy, vol. 5, pp. 237-244, 2012.

[5] P. Moreau, "The emerging role of carfilzomib combination therapy in the management of multiple myeloma," Expert Review of Hematology, vol. 7, no. 2, pp. 265-290, 2014.

[6] J. R. Berenson, J. D. Hilger, O. Yellin et al., "Replacement of bortezomib with carfilzomib for multiple myeloma patients progressing from bortezomib combination therapy," Leukemia, vol. 28, no. 7, pp. 1529-1536, 2014.

[7] P. Neri, N. J. Bahlis, and S. Lonial, "Panobinostat for the treatment of multiple myeloma," Expert Opinion on Investigational Drugs, vol. 21, no. 5, pp. 733-747, 2012.

[8] A. Khot, M. Dickinson, and H. M. Prince, "Panobinostat in lymphoid and myeloid malignancies," Expert Opinion on Investigational Drugs, vol. 22, no. 9, pp. 1211-1223, 2013.

[9] J. F. San-Miguel, P. G. Richardson, A. Günther et al., "Phase Ib study of panobinostat and bortezomib in relapsed or relapsed and refractory multiple myeloma," Journal of Clinical Oncology, vol. 31, no. 29, pp. 3696-3703, 2013.

[10] J. R. Berenson, J. D. Hilger, O. Yellin et al., "A phase 1/2 study of oral panobinostat combined with melphalan for patients with relapsed or refractory multiple myeloma," Annals of Hematology, vol. 93, no. 1, pp. 89-98, 2014. 
[11] P. G. Richardson, R. L. Schlossman, M. Alsina et al., "PANORAMA 2: panobinostat in combination with bortezomib and dexamethasone in patients with relapsed and bortezomib-refractory myeloma," Blood, vol. 122, no. 14, pp. 2331-2337, 2013.

[12] R. Feng, A. Oton, M. Y. Mapara, G. Anderson, C. Belani, and S. Lentzsch, "The histone deacetylase inhibitor, PXD101, potentiates bortezomib-induced anti-multiple myeloma effect by induction of oxidative stress and DNA damage," The British Journal of Haematology, vol. 139, no. 3, pp. 385-397, 2007.

[13] T. Hideshima, J. E. Bradner, J. Wong et al., "Small-molecule inhibition of proteasome and aggresome function induces synergistic antitumor activity in multiple myeloma," Proceedings of the National Academy of Sciences of the United States of America, vol. 102, no. 24, pp. 8567-8572, 2005.

[14] S. Bhalla, S. Balasubramanian, K. David, and et al, "PCI24781 induces caspase and reactive oxygen species-dependent apoptosis through NF- $\kappa \mathrm{B}$ mechanisms and is synergistic with bortezomib in lymphoma cells," Clinical Cancer Research, vol. 15, no. 14, pp. 3354-3365, 2009.

[15] R. Castelli, R. Gualtierotti, N. Orofino, A. Losurdo, S. Gandolfi, and M. Cugno, "Current and emerging treatment options for patients with relapsed myeloma," Clinical Medicine Insights: Oncology, vol. 7, pp. 209-219, 2013.

[16] L. Bastian, J. Hof, M. Pfau et al., "Synergistic activity of bortezomib and HDACi in preclinical models of B-cell precursor acute lymphoblastic leukemia via modulation of p53, PI3K/AKT, and NF- $\kappa$ B," Clinical Cancer Research, vol. 19, no. 6, pp. 1445-1457, 2013.

[17] A. Fribley, Q. Zeng, and C.-Y. Wang, "Proteasome inhibitor PS-341 induces apoptosis through induction of endoplasmic reticulum stress-reactive oxygen species in head and neck squamous cell carcinoma cells," Molecular and Cellular Biology, vol. 24, no. 22, pp. 9695-9704, 2004.

[18] Y.-H. Ling, L. Liebes, Y. Zou, and R. Perez-Soler, "Reactive oxygen species generation and mitochondrial dysfunction in the apoptotic response to bortezomib, a novel proteasome inhibitor, in human H460 Non-small cell lung cancer cells," The Journal of Biological Chemistry, vol. 278, no. 36, pp. 33714-33723, 2003.

[19] R. R. Rosato, S. S. Kolla, S. K. Hock et al., "Histone deacetylase inhibitors activate NF- $\kappa \mathrm{B}$ in human leukemia cells through an ATM/NEMO-related pathway," The Journal of Biological Chemistry, vol. 285, no. 13, pp. 10064-10077, 2010.

[20] J. S. Ungerstedt, Y. Sowa, W.-S. Xu et al., "Role of thioredoxin in the response of normal and transformed cells to histone deacetylase inhibitors," Proceedings of the National Academy of Sciences of the United States of America, vol. 102, no. 3, pp. 673678, 2005.

[21] M. Gao, L. Gao, Y. Tao et al., "Proteasome inhibitor carfilzomib interacts synergistically with histone deacetylase inhibitor vorinostat in Jurkat T-leukemia cells," Acta Biochimica et Biophysica Sinica, vol. 46, no. 6, pp. 484-491, 2014.

[22] G. Dasmahapatra, D. Lembersky, M. P. Son et al., "Carfilzomib interacts synergistically with histone deacetylase inhibitors in mantle cell lymphoma cells in vitro and in vivo," Molecular Cancer Therapeutics, vol. 10, no. 9, pp. 1686-1697, 2011.

[23] X. Wang, J. L. Martindale, Y. Liu, and N. J. Holbrook, "The cellular response to oxidative stress: influences of mitogenactivated protein kinase signalling pathways on cell survival," The Biochemical Journal, vol. 333, no. part 2, pp. 291-300, 1998.
[24] Q.-L. Zhang, L. Wang, Y.-W. Zhang et al., "The proteasome inhibitor bortezomib interacts synergistically with the histone deacetylase inhibitor suberoylanilide hydroxamic acid to induce T-leukemia/lymphoma cells apoptosis," Leukemia, vol. 23, no. 8, pp. 1507-1514, 2009. 

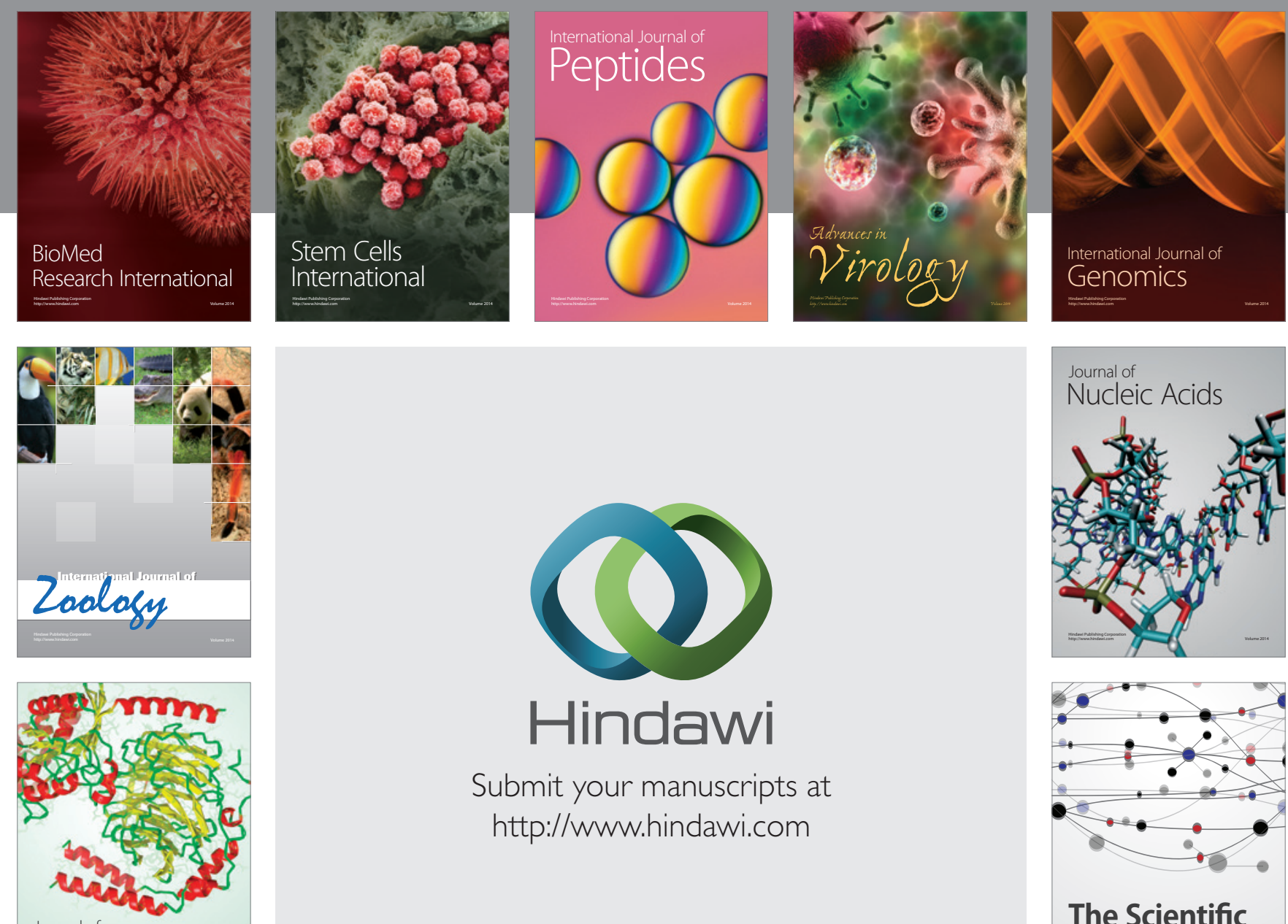

Submit your manuscripts at

http://www.hindawi.com

Journal of
Signal Transduction
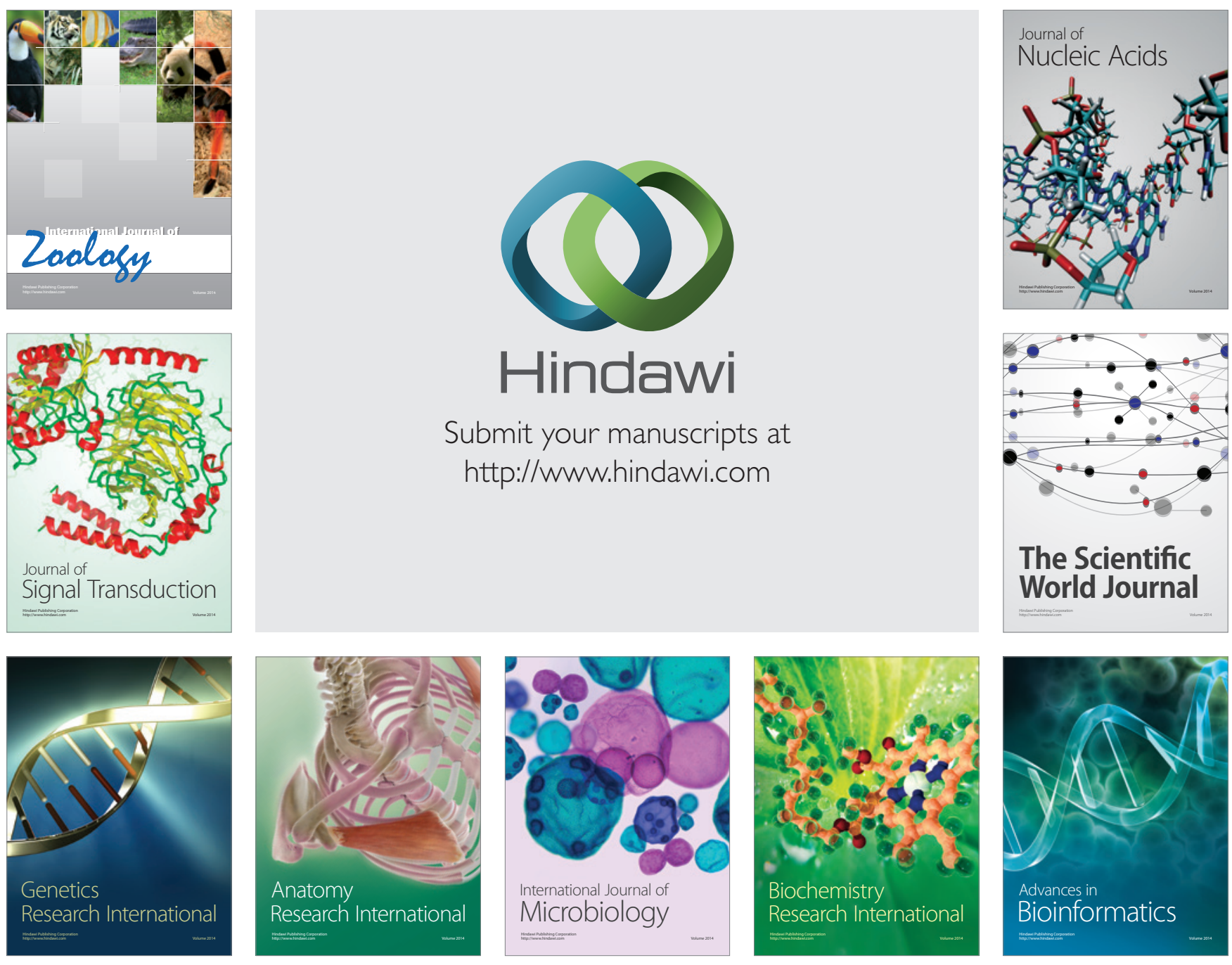

The Scientific World Journal
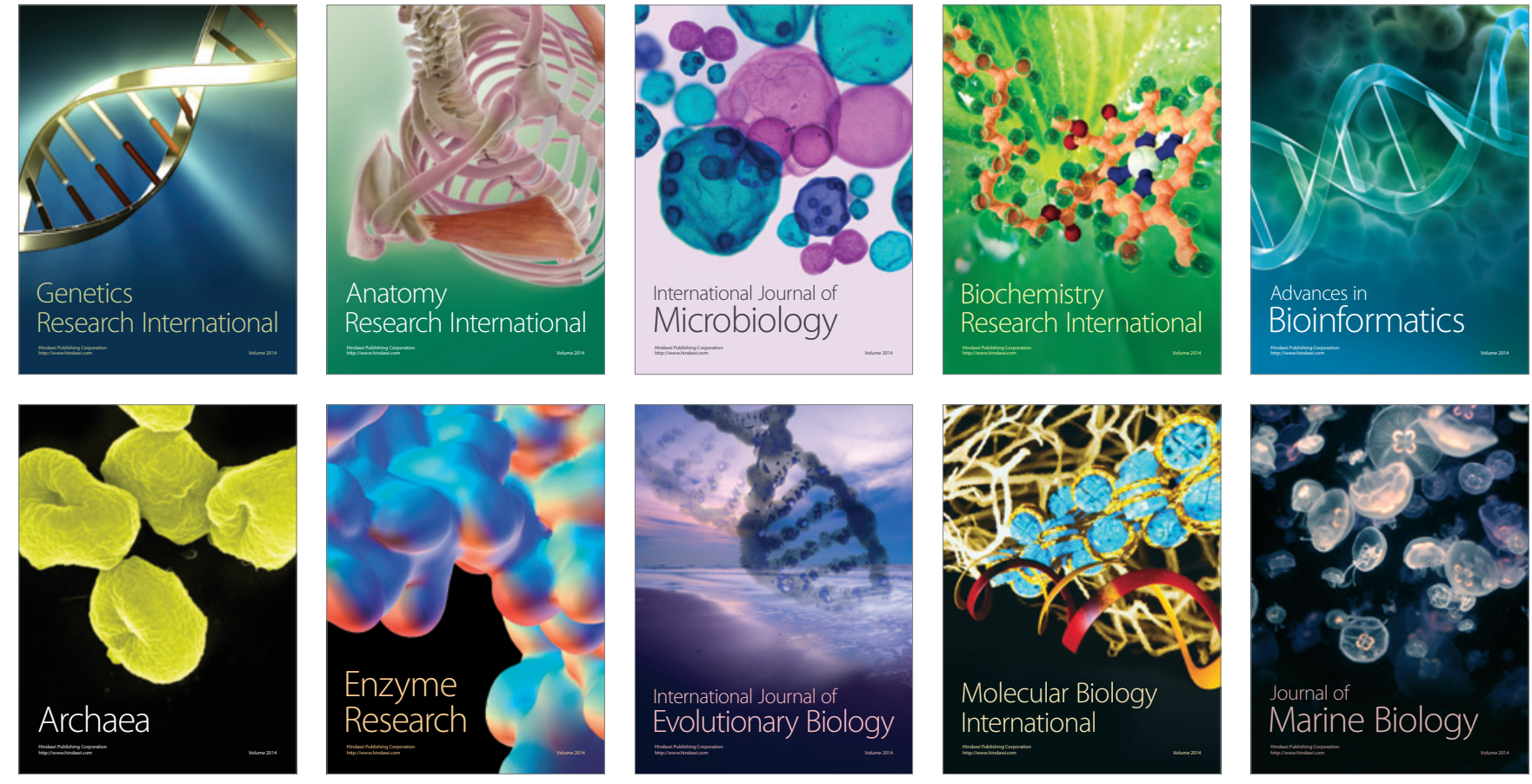